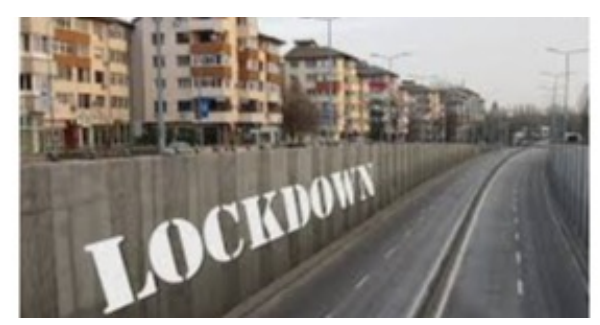

\title{
What Impact has lockdown on SARS-CoV-2/COVID-19 incidence, prevalence and mortality during second wave of pandemic in 2021: - observational analysis of Bihar
}

DR PIYUSH KUMAR ${ }^{1}$

1 B. R. Ambedkar Bihar University

Funding: The author(s) received no specific funding for this work.

Potential competing interests: The author(s) declared that no potential competing interests exist.

\section{Abstract}

Background: My research aimed to assess the impact of lockdown on SARS-CoV-2/COVID-19 pandemic 13 days before lockdown, first and second 13 days during lockdown, and 13 days after the lockdown on the trends in the incidence, prevalence and mortality in the state of Bihar, India, during COVID-19 pandemic.

Methods: The information on the number of cases and deaths due to COVID-19 pandemic in Bihar was obtained from Health Department Bihar, Ministry of Health and Family Welfare, Government of India, and lockdown data were obtained from online websites as well. The impact of lockdown for 13 days before lockdown, first and second 13 days during lockdown, and 13 days after the lockdown on the incidence, prevalence and mortality due to the

COVID-19 pandemic in Bihar was analyzed with Microsoft office and stata 15.1 for windows (64bit) will be used with Microsoft office in next version-2 of article.

Results: The findings showed that except for Incidence /100000/ new death there was a trend toward a decline, and except for Prevalence/100000/confirmed cases from beginning of pandemic all other prevalence have increased. The total and observation period mortality rate due to the COVID-19 pandemic also increased. 
Conclusions: The findings indicate that 13 days after the lockdown, incidence, daily cases of COVID-19 and the growth of the disease showed a declined trend, but there was no significant decline in the prevalence and mortality.

Background: The delivery of health services is of greatest importance and major concern in India particularly populous states like Bihar with imperfect and inadequate resources, lack of modern infrastructure and enormous demand on healthcare structure. The Census 2011 calculated that Bihar has population of 10.41 Crores, an increase from figure of 8.30 Crore in 2001 census. As per 2011 census there were 54,278,157 male and 49,821,295 female respectively. As per projection of census, population of Bihar in 2021 is 13.12 Crore (1)The SARS-CoV-2 pandemic had offered a challenge even for developed healthcare systems around the globe. A sense of panic engrossed the globe due to pandemic and the state of Bihar in India is not exclusion. The insufficient and inadequate healthcare resources including manpower, infrastructure, transportation (ambulance services) etc. have been largely utilized to tackle the situation of pandemic. This shift has tremendous effect of continuing various health programmes running formerly before the pandemic era (2).

The Severe Acute Respiratory Syndrome Coronavirus 2 (SARS-CoV-2) popularly known as COVID-19 pandemic,

first found in Wuhan, China which had spread globally and causing morbidity, mortality as well as huge economic losses. The SARS-CoV-2/ Covid -19 pandemic are not over globally as well as in India and Bihar. The current healthcare system is burdened more with this new diseases added with previous NCD (non communicable diseases) \& CD (communicable diseases). The First human case of this global pandemic was reported from Wuhan city of China in December 2019 (3). The first case ofcovid-19 in India was found in January 2020 and Bihar reported first COVID-19 case from Munger on 22 March 2020, a 38-year-old tested positive for COVID-19, he was also the first victim.

As of June 17, 2021, 07:33 GMT, covid-19 has involved 220 countries and has infected 177, 819, 445, people with a mortality of 3,849,051 deaths (4). The median incubation period for COVID-19 is usually 5.1 days, and may be up to 14 days. The incubation period of COVID-19 is very significant in establishing lockdown, monitoring, surveillance and control of the disease spread. The high contagious nature of COVID-19 has led to panic situation across the globe stopping entry and exit across different boundaries and even upto lowest administrative levels by implications of containment zones. The world-wide population has been under lockdown and quarantined in their homes at some point. The lockdown and quarantine methods have been implemented by many nations and states to control the spread of covid-19.

\section{The lockdown order in Bihar issued by Home Department dated 04/05/2021 announced} lockdown from 05/05/2021 to 15/05/2021 and then extended upto 1 June, 2021, includes several restrictions such as isolation at homes, travel restrictions, and termination of all public events etc (5). The lockdown strategies in Bihar have been enforced like all over the world in order to prevent the COVID-19 infection from spreading even further. On comparing the pattern of transmission rates observed in few 
countries at posterior estimated change points, it is found that partial implementation of lockdown (in the United States), delayed planning in lockdown (Russia, United Kingdom, and France), and inadequate implementation of the lockdown (in India and Italy) were found to be mainly responsible for the spread of covid-19 infections (6).

Vaccines are known to effectively prevent a COVID-19 infection and reduce morbidity-mortality but there are multiple factors and obstacles in running smoothly the vaccination programme such as frequent change and unavailability of vaccines, guidelines, policies, interdisciplinary conflicts of medical sciences, mistrust, evil propaganda over Government data, lack of communication and health promotion in rural areas of India (7). Hence in current scenario of Bihar and most of states in India, public health measures such as lockdown, masks, quarantine, and social distancing appear to be the only ways to control the outbreak. Lockdown and quarantine can either be applied on a voluntary basis, or if seems necessary, can be legally forced by the authorities, and may be implemented at individual or community levels. The home quarantine, when scientifically and adequately applied and exercised according to covid-19 principles, guidelines, protocols and practices, can be quite effective for preventing the spread of covid-19 diseases. Globally, many countries have imposed a lockdown, quarantine period for over several days to months for this purpose. There are great economic concerns as well as question on the effectiveness and risks of longterm implementation of a lock-down and or quarantine. Keeping in view the rapid spread of COVID-19 cases and rise in mortality and morbidity in Bihar, the present study aimed to investigate the impact of lockdowns for 13 days before, first and second 13 days during lockdown and 13 days afterward on international epidemiological trends in the prevalence and mortality of COVID-19 cases.

\section{Materials and methods}

The present observational study was conducted by the author during the period of May-June 2021. The data on the trends in the incidence, prevalence and mortality due to COVID-19 outbreak in Bihar were collected on daily basis from Health Department, Bihar as well as Ministry of Health and Family Welfare, Government of India also matched with online sources available in Google search. The daily reports on COVID-19 published by the Health Department, Bihar as well as Ministry of Health and Family Welfare, Government of India through website, twitter etc. were care-fully reviewed and data were collected. The lockdown data were obtained time to time from concerned authorities. The population data of Bihar was obtained from the census2011 publications. Data of all the 38 districts of Bihar have been collected and analyzed as well as calculation for incidence, prevalence and mortality was done and presented as table( see table 1,2,3,4,5,6,7,8 and graph 1 ) and graph in this article.

The growth factor I considered is as a ratio equals to cases on last day of each of four observations divided by cases on the first day of observation (see table-9). A growth factor of more than 1.0 indicates an increasing pattern of prevalence, whereas values between below 1.0 show a declining pattern. A positive growth factor indicates exponential growth in the number of cases and a negative growth factor for the period indicates exponential decay in the number of new cases. A negative growth rate per observation period means an epidemic is coming under control because in that case, the number of new cases each 
day will be decreasing and heading in a direction toward no new cases in a day. The collected data were properly recorded and analyzed because the pandemic has been changing numbers daily. I analyzed the impact of lockdown on the growth factor, incidence, prevalence and mortality due to COVID-19 outbreak in Bihar, India by establishing an calculating-analyzing association between the numbers, 13 days before, 26 days during (divided into two equal parts 13 days each, termed first 13 and second 13) and 13 days after the end of the lockdown period on 01/06/2021.

\section{Statistical analysis}

The data were recorded, calculated and analyzed with Microsoft office in this version 1 (in next version two stata software added with this for analysis), and the output-results were expressed in numbers and percentages presented in tables $1,2,3,4,5,6,7,8,9,10$. The average of confirmed new cases/new active cases/new cured cases/new death, prevalence and mortality of cases were calculated. The growth factor, by which quantity multiplies itself over time; here $13^{\text {th }}$ day cases divided by cases on the first day was calculated.

\section{Results}

The total number of laboratory-confirmed cases / active cases/ cured/discharged/ and deaths due to covid2019 pandemic 13 days before lockdown are presented in Table 1. The total number of laboratoryConfirmed New cases / New Active Cases/ New Cured/ New Discharged/ and New deaths due to Covid-2019 pandemic 13 days before lockdown are presented in Table 2.

The impact of the lockdown on the epidemiological trends of covid-19 is presented in Tables 3, 4, 5, and 6. The epidemiological trends after lockdown are presented in tables 7 and 8. Table 9 shows the Growth Factor for Number of laboratory-Confirmed New cases / New Active Cases/ New Cured/ New Discharged/ and New deaths/ Confirmed cases / Active Cases/ Cured/Discharged/ and deaths due to Covid-2019 pandemic. Table 10 presents the Prevalence/Incidence/Mortality of Number of laboratory-Confirmed New cases / New Active Cases/ New Cured/ New Discharged/ and New deaths/ Confirmed cases / Active Cases/ Cured/Discharged/ and deaths due to Covid-2019 pandemic. Table 11 presents the average number of confirmed new cases/new active cases/new cured cases/new death, at 13 days before, 26 days during, and 13 days after the lockdown in Bihar, India and correlations were established.

Table 1 Bihar- total number of laboratory-confirmed cases / active cases/ cured/discharged/ and deaths due to covid-2019 pandemic 13 days before lockdown 


\begin{tabular}{|c|c|c|c|c|c|}
\hline Date & Region & Confirmed Cases & Active Cases & Cured/Discharged & Death \\
\hline $22 / 04 / 2021$ & Bihar & 354281 & 63747 & 288637 & 1897 \\
\hline $23 / 04 / 2021$ & Bihar & 365770 & 69869 & 293945 & 1956 \\
\hline $24 / 04 / 2021$ & Bihar & 378442 & 76420 & 300012 & 2010 \\
\hline $25 / 04 / 2021$ & Bihar & 390801 & 81961 & 306753 & 2087 \\
\hline $26 / 04 / 2021$ & Bihar & 403596 & 87155 & 314286 & 2155 \\
\hline $27 / 04 / 2021$ & Bihar & 415397 & 89661 & 323514 & 2222 \\
\hline $28 / 04 / 2021$ & Bihar & 428001 & 94276 & 331418 & 2307 \\
\hline 29/04/2021 & Bihar & 441375 & 98748 & 340236 & 2391 \\
\hline $30 / 04 / 2021$ & Bihar & 454464 & 100822 & 351162 & 2480 \\
\hline $1 / 5 / 2021$ & Bihar & 470317 & 105401 & 362356 & 2560 \\
\hline $2 / 5 / 2021$ & Bihar & 484106 & 108203 & 373261 & 2642 \\
\hline $3 / 5 / 2021$ & Bihar & 497640 & 109946 & 384955 & 2739 \\
\hline $4 / 5 / 2021$ & Bihar & 509047 & 107668 & 398558 & 2821 \\
\hline Total & & 509047 & 107668 & 398558 & 2821 \\
\hline
\end{tabular}

Table 2 Bihar- Number of laboratory-Confirmed New cases / NewActive Cases/ New Cured/ New Discharged/ and New deaths due to Covid-2019 pandemic 13 days before lockdown

\begin{tabular}{|c|c|c|c|c|c|}
\hline Date & Confirmed New Cases & New Active Cases & $\begin{array}{l}\text { New } \\
\text { Cured/Discharged }\end{array}$ & New Death & $\begin{array}{l}\text { Population-2011 } \\
\text { Census }\end{array}$ \\
\hline $22 / 04 / 2021$ & 12222 & 7392 & 4774 & 56 & 104099452 \\
\hline $23 / 04 / 2021$ & 11489 & 6122 & 5308 & 59 & 104099452 \\
\hline $24 / 04 / 2021$ & 12672 & 6551 & 6067 & 54 & 104099452 \\
\hline $25 / 04 / 2021$ & 12359 & 5541 & 6741 & 77 & 104099452 \\
\hline $26 / 04 / 2021$ & 12795 & 5194 & 7533 & 68 & 104099452 \\
\hline $27 / 04 / 2021$ & 11801 & 2506 & 9228 & 67 & 104099452 \\
\hline $28 / 04 / 2021$ & 12604 & 4615 & 7904 & 85 & 104099452 \\
\hline 29/04/2021 & 13374 & 4472 & 8818 & 84 & 104099452 \\
\hline $30 / 04 / 2021$ & 13089 & 2074 & 10926 & 89 & 104099452 \\
\hline $1 / 5 / 2021$ & 15853 & 4579 & 11194 & 80 & 104099452 \\
\hline $2 / 5 / 2021$ & 13789 & 2802 & 10905 & 82 & 104099452 \\
\hline $3 / 5 / 2021$ & 13534 & 1743 & 11694 & 97 & 104099452 \\
\hline $4 / 5 / 2021$ & 11407 & -2278 & 13603 & 82 & 104099452 \\
\hline Total & 166988 & 51313 & 114695 & 980 & \\
\hline
\end{tabular}

Table 3 Bihar- Total Number of laboratory-Confirmed cases / Active Cases/

Cured/Discharged/ and deaths due to Covid-2019 pandemic-first 13 days of lockdown 


\begin{tabular}{|c|c|c|c|c|c|}
\hline Date & Region & Confirmed Cases & Active Cases & Cured/Discharged & Death \\
\hline $5 / 5 / 2021$ & Bihar & 523841 & 110431 & 410484 & 2926 \\
\hline $6 / 5 / 2021$ & Bihar & 538677 & 113480 & 422210 & 2987 \\
\hline $7 / 5 / 2021$ & Bihar & 553803 & 115152 & 435574 & 3077 \\
\hline $8 / 5 / 2021$ & Bihar & 567269 & 115067 & 449063 & 3139 \\
\hline $9 / 5 / 2021$ & Bihar & 580217 & 112977 & 464025 & 3215 \\
\hline $10 / 5 / 2021$ & Bihar & 591476 & 110805 & 477389 & 3282 \\
\hline $11 / 5 / 2021$ & Bihar & 601650 & 105104 & 493189 & 3357 \\
\hline $12 / 5 / 2021$ & Bihar & 612570 & 102100 & 507041 & 3429 \\
\hline $13 / 05 / 2021$ & Bihar & 622433 & 99624 & 519306 & 3503 \\
\hline $14 / 05 / 2021$ & Bihar & 622433 & 99624 & 519306 & 3503 \\
\hline $15 / 05 / 2021$ & Bihar & 630185 & 96278 & 530314 & 3593 \\
\hline $16 / 05 / 2021$ & Bihar & 645015 & 82487 & 558785 & 3743 \\
\hline $17 / 05 / 2021$ & Bihar & 651909 & 75090 & 572987 & 3832 \\
\hline Total & & 651909 & 75090 & 572987 & 3832 \\
\hline
\end{tabular}

Table 4 Bihar- Number of laboratory-Confirmed New cases / New Active Cases/ New Cured/ New Discharged/ and New deaths due to Covid-2019 pandemic-first 13 days of lockdown

\begin{tabular}{|c|c|c|c|c|c|}
\hline Date & Confirmed New Cases & New Active Cases & $\begin{array}{l}\text { New } \\
\text { Cured/Discharged }\end{array}$ & New Death & $\begin{array}{l}\text { Population-2011 } \\
\text { Census }\end{array}$ \\
\hline $5 / 5 / 2021$ & 14794 & 2763 & 11926 & 105 & 104099452 \\
\hline $6 / 5 / 2021$ & 14836 & 3049 & 11726 & 61 & 104099452 \\
\hline $7 / 5 / 2021$ & 15126 & 1672 & 13364 & 90 & 104099452 \\
\hline $8 / 5 / 2021$ & 13466 & -85 & 13489 & 62 & 104099452 \\
\hline $9 / 5 / 2021$ & 12948 & -2090 & 14962 & 76 & 104099452 \\
\hline $10 / 5 / 2021$ & 11259 & -2172 & 13364 & 67 & 104099452 \\
\hline $11 / 5 / 2021$ & 10174 & -5701 & 15800 & 75 & 104099452 \\
\hline $12 / 5 / 2021$ & 10920 & -3004 & 13852 & 72 & 104099452 \\
\hline $13 / 05 / 2021$ & 9863 & -2476 & 12265 & 74 & 104099452 \\
\hline $14 / 05 / 2021$ & 0 & 0 & 0 & 0 & 104099452 \\
\hline $15 / 05 / 2021$ & 7752 & -3346 & 11008 & 90 & 104099452 \\
\hline $16 / 05 / 2021$ & 14830 & -13791 & 28471 & 150 & 104099452 \\
\hline 17/05/2021 & 6894 & -7397 & 14202 & 89 & 104099452 \\
\hline Total & 142862 & -32578 & 174429 & 1011 & \\
\hline
\end{tabular}

Table 5 Bihar- Total Number of laboratory-Confirmed cases / Active Cases/

Cured/Discharged/ and deaths due to Covid-2019 pandemic-second 13 days of lockdown 


\begin{tabular}{|c|c|c|c|c|c|}
\hline Date & Region & Confirmed Cases & Active Cases & Cured/Discharged & Death \\
\hline $18 / 05 / 2021$ & Bihar & 657829 & 69698 & 584203 & 3928 \\
\hline 19/05/2021 & Bihar & 664115 & 64699 & 595377 & 4039 \\
\hline 20/05/2021 & Bihar & 670174 & 58611 & 607420 & 4143 \\
\hline 21/05/2021 & Bihar & 676045 & 54407 & 617397 & 4241 \\
\hline $22 / 05 / 2021$ & Bihar & 681199 & 49312 & 627548 & 4339 \\
\hline 23/05/2021 & Bihar & 685574 & 44908 & 636224 & 4442 \\
\hline 24/05/2021 & Bihar & 689576 & 40692 & 644335 & 4549 \\
\hline $25 / 05 / 2021$ & Bihar & 692420 & 37943 & 649835 & 4642 \\
\hline 26/05/2021 & Bihar & 695726 & 35130 & 655850 & 4746 \\
\hline 27/05/2021 & Bihar & 698329 & 30993 & 662491 & 4845 \\
\hline 28/05/2021 & Bihar & 700897 & 28448 & 667506 & 4943 \\
\hline $30 / 05 / 2021$ & Bihar & 704173 & 21085 & 678036 & 5052 \\
\hline $31 / 05 / 2021$ & Bihar & 705648 & 18378 & 682166 & 5104 \\
\hline Total & & 705648 & 18378 & 682166 & 5104 \\
\hline
\end{tabular}

Table 6 Bihar- Number of laboratory-Confirmed New cases / New Active Cases/ New Cured/ New Discharged/ and New deaths due to Covid-2019 pandemic-second 13 days of lockdown

\begin{tabular}{|c|c|c|c|c|c|}
\hline Date & Confirmed New Cases & New Active Cases & $\begin{array}{l}\text { New } \\
\text { Cured/Discharged }\end{array}$ & New Death & $\begin{array}{l}\text { Population-2011 } \\
\text { Census }\end{array}$ \\
\hline $18 / 05 / 2021$ & 5920 & -5392 & 11216 & 96 & 104099452 \\
\hline $19 / 05 / 2021$ & 6286 & -4999 & 11174 & 111 & 104099452 \\
\hline $20 / 05 / 2021$ & 6059 & -6088 & 12043 & 104 & 104099452 \\
\hline $21 / 05 / 2021$ & 5871 & -4204 & 9977 & 98 & 104099452 \\
\hline $22 / 05 / 2021$ & 5154 & -5095 & 10151 & 98 & 104099452 \\
\hline $23 / 05 / 2021$ & 4375 & -4404 & 8676 & 103 & 104099452 \\
\hline $24 / 05 / 2021$ & 4002 & -4216 & 8111 & 107 & 104099452 \\
\hline $25 / 05 / 2021$ & 2844 & -2749 & 5500 & 93 & 104099452 \\
\hline $26 / 05 / 2021$ & 3306 & -2813 & 6015 & 104 & 104099452 \\
\hline $27 / 05 / 2021$ & 2603 & -4137 & 6641 & 99 & 104099452 \\
\hline $28 / 05 / 2021$ & 2568 & -2545 & 5015 & 98 & 104099452 \\
\hline $30 / 05 / 2021$ & 3276 & -7363 & 10530 & 109 & 104099452 \\
\hline $31 / 05 / 2021$ & 1475 & -2707 & 4130 & 52 & 104099452 \\
\hline Total & 53739 & -56712 & 109179 & 1272 & \\
\hline
\end{tabular}


Table 7 Bihar- Total Number of laboratory-Confirmed cases / Active Cases/

Cured/Discharged/ and deaths due to Covid-2019 pandemic-first 13 days after lockdown

\begin{tabular}{|c|c|c|c|c|c|}
\hline Date & Region & Confirmed Cases & Active Cases & Cured/Discharged & Death \\
\hline $2 / 6 / 2021$ & Bihar & 707935 & 14251 & 688462 & 5222 \\
\hline $3 / 6 / 2021$ & Bihar & 709093 & 12591 & 691234 & 5268 \\
\hline $4 / 6 / 2021$ & Bihar & 710199 & 11431 & 693472 & 5296 \\
\hline $5 / 6 / 2021$ & Bihar & 711190 & 10309 & 695562 & 5319 \\
\hline $6 / 6 / 2021$ & Bihar & 712197 & 9628 & 697229 & 5340 \\
\hline $7 / 6 / 2021$ & Bihar & 713117 & 8708 & 699028 & 5381 \\
\hline $8 / 6 / 2021$ & Bihar & 713879 & 8231 & 700224 & 5424 \\
\hline 9/6/2021 & Bihar & 714590 & 7898 & 701234 & 5458 \\
\hline $10 / 6 / 2021$ & Bihar & 715179 & 4516 & 701234 & 9429 \\
\hline $11 / 6 / 2021$ & Bihar & 715730 & 5044 & 701234 & 9452 \\
\hline $12 / 6 / 2021$ & Bihar & 716296 & 5596 & 701234 & 9466 \\
\hline $13 / 06 / 2021$ & Bihar & 716728 & 5701 & 701543 & 9484 \\
\hline $14 / 06 / 2021$ & Bihar & 717215 & 5312 & 702411 & 9492 \\
\hline Total & & 717215 & 5312 & 702411 & 9492 \\
\hline
\end{tabular}

Table 8 Bihar- Number of laboratory-Confirmed New cases / New Active Cases/ New Cured/ New Discharged/ and New deaths/ due to Covid-2019 pandemic- first 13 days after lockdown

\begin{tabular}{|c|c|c|c|c|c|}
\hline Date & Confirmed New Cases & New Active Cases & $\begin{array}{l}\text { New } \\
\text { Cured/Discharged }\end{array}$ & New Death & $\begin{array}{l}\text { Population-2011 } \\
\text { Census }\end{array}$ \\
\hline $2 / 6 / 2021$ & 2287 & -4127 & 6296 & 118 & 104099452 \\
\hline $3 / 6 / 2021$ & 1158 & -1660 & 2772 & 46 & 104099452 \\
\hline 4/6/2021 & 1106 & -1160 & 2238 & 28 & 104099452 \\
\hline $5 / 6 / 2021$ & 991 & -1122 & 2090 & 23 & 104099452 \\
\hline $6 / 6 / 2021$ & 1007 & -681 & 1667 & 21 & 104099452 \\
\hline $7 / 6 / 2021$ & 920 & -920 & 1799 & 41 & 104099452 \\
\hline $8 / 6 / 2021$ & 762 & -477 & 1196 & 43 & 104099452 \\
\hline $9 / 6 / 2021$ & 711 & -333 & 1010 & 34 & 104099452 \\
\hline $10 / 6 / 2021$ & 589 & -3382 & $\mathbf{0}$ & 3971 & 104099452 \\
\hline $11 / 6 / 2021$ & 551 & 528 & $\mathbf{0}$ & 23 & 104099452 \\
\hline $12 / 6 / 2021$ & 566 & 552 & $\mathbf{0}$ & 14 & 104099452 \\
\hline $13 / 06 / 2021$ & 432 & 105 & 309 & 18 & 104099452 \\
\hline $14 / 06 / 2021$ & 487 & -389 & 868 & 8 & 104099452 \\
\hline Total & 11567 & -13066 & 20245 & 438 & \\
\hline
\end{tabular}

Table 9 Bihar-Growth Factor- Number of laboratory-Confirmed New cases / New Active Cases/ New Cured/ New Discharged/ and New deaths/ Confirmed cases / Active Cases/ Cured/Discharged/ and deaths due to Covid-2019 pandemic 


\begin{tabular}{|c|c|c|c|c|}
\hline Prevalence/Incidence/Mortality & $\begin{array}{l}\text { Growth Factor First } 13 \\
\text { days before lockdown }\end{array}$ & $\begin{array}{l}\text { Growth Factor First } \\
13 \text { day of lockdown }\end{array}$ & $\begin{array}{l}\text { Growth Factor } \\
\text { Second } 13 \text { day of } \\
\text { lockdown }\end{array}$ & $\begin{array}{l}\text { Growth Factor First } \\
13 \text { day after lockdown }\end{array}$ \\
\hline confirmed new cases & 0.933316969 & 0.46599973 & 0.249155405 & 0.21294272 \\
\hline confirmed new active cases & -0.308170996 & -2.677162505 & 0.502040059 & 0.09425733 \\
\hline new discharged cases & 2.849392543 & 1.190843535 & 0.368223966 & 0.137865311 \\
\hline new death & 1.464285714 & 0.847619048 & 0.541666667 & 0.06779661 \\
\hline $\begin{array}{l}\text { confirmed cases basis- } \\
\text { Observation on } 05 / 05 / 20\end{array}$ & 1.436845329 & 1.244478764 & 1.072692143 & 1.013108548 \\
\hline $\begin{array}{l}\text { active cases basis- } \\
\text { Observation on } 05 / 05 / 20\end{array}$ & 1.688989286 & 0.679972109 & 0.26368045 & 0.372745772 \\
\hline $\begin{array}{l}\text { discharged cases basis- } \\
\text { Observation on } 05 / 05 / 20\end{array}$ & 1.380827822 & 1.395881447 & 1.167686575 & 1.020261104 \\
\hline $\begin{array}{l}\text { Prevalence basis- } \\
\text { Observation on } 05 / 05 / 20\end{array}$ & 1.487085 & 1.309638 & 1.299389 & 1.817694 \\
\hline $\begin{array}{l}\text { Mortality basis- } \\
\text { Observation on } 05 / 05 / 20\end{array}$ & 1.487085 & 1.309638 & 1.299389 & 1.817694 \\
\hline $\begin{array}{l}\text { Total Mortality basis- } \\
\text { Observation on } 05 / 05 / 20\end{array}$ & 1.464285714 & 0.847619048 & 0.541666667 & 0.06779661 \\
\hline
\end{tabular}

Table 10 Bihar- Prevalence/Incidence/Mortality - Number of laboratory-Confirmed New cases / New Active Cases/ New Cured/ New Discharged/ and New deaths/ Confirmed cases / Active Cases/Cured/Discharged/ and deaths due to Covid-2019 pandemic 


\begin{tabular}{|c|c|c|c|c|c|}
\hline Prevalence/Incidence/Mortality & $\begin{array}{l}\text { First } 13 \text { days } \\
\text { before lockdown }\end{array}$ & $\begin{array}{l}\text { First } 13 \text { day } \\
\text { of lockdown }\end{array}$ & $\begin{array}{l}\text { Second } 13 \text { day } \\
\text { of lockdown }\end{array}$ & $\begin{array}{l}\text { First } 13 \text { day } \\
\text { after lockdown }\end{array}$ & $\begin{array}{l}\text { Final increase } \\
\text { or decrease }\end{array}$ \\
\hline $\begin{array}{l}\text { Incidence/100000/ confirmed new } \\
\text { cases }\end{array}$ & 160.4119876 & 137.2360731 & 51.62275014 & 11.11148981 & decrease \\
\hline $\begin{array}{l}\text { Incidence } / 100000 / \text { confirmed new } \\
\text { active cases }\end{array}$ & 49.29228638 & -31.29507348 & -54.47867295 & -12.55145896 & decrease \\
\hline $\begin{array}{l}\text { Incidence /100000/ new discharged } \\
\text { cases }\end{array}$ & 110.1782937 & 167.5599599 & 104.8795146 & 19.44774887 & decrease \\
\hline Incidence /100000/ new death & 0.941407453 & 0.971186669 & 1.22190845 & 4.215199903 & increase \\
\hline $\begin{array}{l}\text { Prevalence } / 100000 / \text { confirmed cases } \\
\text { from beginning of pandemic }\end{array}$ & 489.000653 & 626.236726 & 677.8594761 & 688.970966 & increase \\
\hline $\begin{array}{l}\text { Prevalence/100000/active cases } \\
\text { from beginning of pandemic }\end{array}$ & 103.428018 & 72.13294456 & 17.65427161 & 5.102812645 & decrease \\
\hline $\begin{array}{l}\text { Prevalence } / 100000 / \text { discharged cases } \\
\text { from beginning of pandemic }\end{array}$ & 382.8627263 & 550.4226862 & 655.3022008 & 674.7499497 & increase \\
\hline $\begin{array}{l}\text { Prevalence } / 100000 / \text { death cases } \\
\text { from beginning of pandemic }\end{array}$ & 2.709909 & 3.681095 & 4.903004 & 9.118204 & increase \\
\hline $\begin{array}{l}\text { Mortality Rate/1000-Total from } \\
\text { beginning of pandemic }\end{array}$ & 0.027099 & 0.036811 & 0.04903 & 0.091182 & increase \\
\hline Mortality Rate/1000-13days & 0.009414075 & 0.009711867 & 0.012219084 & 0.042151999 & increase \\
\hline
\end{tabular}

Table 11- Average of confirmed new cases/new active cases/new cured cases/new death

\begin{tabular}{|c|c|c|c|c|}
\hline Period & $\begin{array}{l}\text { Average Confirmed New } \\
\text { Cases }\end{array}$ & $\begin{array}{l}\text { Average New Active } \\
\text { Cases }\end{array}$ & $\begin{array}{l}\text { Average New } \\
\text { Cured/Discharged }\end{array}$ & $\begin{array}{l}\text { Average New } \\
\text { Death }\end{array}$ \\
\hline $\begin{array}{l}\text { First } 13 \text { days before } \\
\text { lockdown }\end{array}$ & 12845.23077 & 3947.153846 & 8822.692308 & 75.38461538 \\
\hline First 13 day of lockdown & 10989.38462 & -2506 & 13417.61538 & 77.76923077 \\
\hline $\begin{array}{l}\text { Second } 13 \text { day of } \\
\text { lockdown }\end{array}$ & 4133.769231 & -4362.461538 & 8398.384615 & $\mathbf{9 7 . 8 4 6 1 5 3 8 5}$ \\
\hline $\begin{array}{l}\text { First } 13 \text { day after } \\
\text { lockdown }\end{array}$ & 889.7692308 & -1005.076923 & 1557.307692 & 337.5384615 \\
\hline
\end{tabular}

The average calculation shows that majority of these coronavirus new cases/new active cases/new discharged cases, was reported during the period 13 days before lockdown whereas average maximum new death were reported during 13 day period after lockdown(Table 11).

Regarding the impact of lockdown on the prevalence and mortality of the COVID-19 outbreak in Bihar, India, I found that 13 days after the lockdown there was no decline in the mean prevalence and the mean number

of daily deaths due to COVID-19 compared to 13 days before and 13 days during the lockdown 
(Tables 10). However, the growth rate in the number of new daily cases of COVID-19 per 13 day (table9) and growth rate in the

number of new deaths per13 day attributed to COVID-19 each showed a positive but falling trend 13 days after the lockdown period in Bihar, India. This data show a negative growth factor per 13 day during the 13 days following the lockdown for new daily cases and for new deaths per day. The change in growth rates

and growth rates per 13 day are expressed as: pre-lockdown vs. lockdown and after lockdown periods. post-lockdown time periods, there was a declining rate of change per day for most except average new death. Regarding the mean prevalence of COVID-19 cases 13 days before, 26 days during and 13 days after lockdown, I found that the mean numbers of cases increased and there was no important impact of lockdown on the prevalence of COVID-19 cases (Tables 10). I calculated growth rate for new cases of COVID-19. The mean growth rate for number of new cases on a 13day basis was 0.21294272 and for new mortality rate was 0.06779661 (table 9 ). It was found that, 13 days after the lockdown, the growth factor of the number of new daily cases decreased and the growth factor of new daily deaths was increased after the lockdown period (table 9).

\section{Discussion}

The COVID-19 pandemic is a major public health problem which has infected millions of people worldwide. The idea of lockdown is associated with the incubation period of COVID-19, which is from 1-14 days (3). The lockdown methods have been implemented in many countries to control the spread of COVID-19 when other measures fail to achieve desired effect. For keeping an epidemic under control we must first control the rate of growth per day to become negative. In this study, I observed the impact of lockdown 13 days before(for comparison), two period of 13 days during and 13 days after(for comparison) lockdown on the epidemiological basis in the growth factor, incidence, prevalence and mortality because of the outbreak of novel coronavirus SARS-COV-2 in Bihar, India. I observed that 13 days after the Bihar lockdown there was no significant decline in the mean prevalence and mean mortality rate due to COVID-19 compared to 13 days before and 13-13(two observation) days during the lockdown in Bihar, India. However, daily cases of COVID-19 and growth rates showed declining trends by the end of the lockdown and after the lockdown period, leading to a critically important negative growth rate by end of the lockdown period for both new daily cases. This negative growth rate per day shows that from a public health perspective, the lockdown had a positive effect on the pandemic . However, the growth rate never fell immediately following the lockdown and moreover lockdown cannot be enforced for a longer time due to economic and various other reasons of public concern, so the lockdown is not the only way to control the pandemic. However when all measures fail the government is forced to impose new lockdowns and encourage residents to isolate themselves in their houses for saving lives.

Through this article I recommend that along with other public health measures, lockdown should be enforced at 
an early stage to prevent the COVID-19 infection from spreading to a large section of population causing increased morbidity and mortality as well as overburden on the health system. The study also demonstrates evidence that

lockdown measures are consistently beneficial. My study observation showed that the lockdown was beneficial in decreasing the rate of growth. The concept of a lockdown is theoretically very attractive because it minimizes the number of people exposed to contagious patients and therefore fewer people will be susceptible to getting infected but practically in poor states like Bihar it's like a tragedy for people who are not able to afford food if they are not getting works on daily basis.

A lockdown may play a significant role when vaccination or prophylactic treatment is not available, as seen in

the case with COVID-19 pandemic. In this research, I observed and analyzed the impact of 13 days before 26 days during and 13 days after lockdown on the prevalence and other epidemiology of COVID-19 cases in Bihar, India. My study findings support hypothesis that lockdown will significantly decrease the number of cases.

I have done this study in a different way and new people in public health may have little problem in understanding my observations. In many countries especially the developing countries long-term lockdown is not sustainable and practically possible as it has various mental, social, psychological and economic impacts. Future lockdown strategy should think of optimizing behavior, health promotion such as social distancing and mask wearing associated with social and cultural factors that can help in controlling the COVID-19 pandemic, because lockdown alone will not be effective if people will not adhere to this policy.

\section{Study strengths and limitations}

This is the first article in the literature, to my knowledge, that have investigated the impact of a lockdown on

epidemiological trends of prevalence and mortality of the COVID-19 pandemic in Bihar, India. During the COVID-19 pandemic, to date, several mathematical modeling-based reviews/articles have been published to hypothesize the impact of a lockdown on the prevalence of COVID-19 cases. This is the first study, which analyzed the impact of 13 days before, 13-13 days during and 13 days after lockdown on the prevalence trends of COVID-19 in Bihar especially point prevalence.

One of the peculiar strength is that the study data were gathered using reliable accredited sources including Government Health Department. I have analyzed the growth factor and the growth rate per 13 day, which are

exceptional and totally new my idea to determine the epidemiological trends of a pandemic. A limitation is that I am unable to investigate confounding factors and bias such as how much people varies in: (1) adherence to lockdown, (2) adoption of protocols and guidelines of social distancing, (3) practice of health hygienic guidelines and (4) experience disease testing systems of nearest health centers.

\section{Conclusions}

My research shows that 13 days after lockdown there was no significant decline in the mean prevalence 
and mean mortality rate due to novel coronavirus SARS-COV 2 compared to 13 days before and 13-13 days during the lockdown in Bihar, India. The study found that daily cases of SARS-COV-2 patients, and the growth factor results

declined and the growth rate per day both declined to an impressive negative level in the case of the growth rate.

These findings may be useful for policy-makers who are thinking of further lockdowns to control the spread of the COVID-19 pandemic. Future lockdown policies should better work for optimizing health behavior like social distancing and mask wearing associated with cultural factors that can halt spreading the COVID-19 pandemic, because lockdown will not be effective if people will not adhere to this policy, guidelines and protocols added with negative impacts of lockdown on livelihood in poor states like Bihar.

\section{'Declarations':}

-This paper has not been previously published and is not currently under consideration by another journal. The document is Microsoft word with English (United States) language \& 4704 words Total.

- Ethics approval and consent to participate: Not applicable. This study has not involved any human or animals in real or for experiments. The data on the prevalence and mortality due to COVID-19 pandemic were taken from the Health Department, Bihar and other organizations which are also available online, hence ethical approval was not required.

-Consent for publication: Not applicable

-Availability of data and materials: The data \& materials for study are mentioned in article and available as reference.

-Conflicts of Interest/ Competing Interest: There are no conflicts / competing of interest

- Funding-Self sponsored. No aid taken from individual or agency etc.

- Authors' contributions: The whole work is solely done by the Author - Dr Piyush Kumar, M.B.B.S. - Sri Krishna Medical College, EMOC- General Medical Officer- Bihar Health Services- Government of Bihar, India.

- Acknowledgements- I am thankful to Advocate Anupama my wife for cooperation.

- Author information: The author is currently working as general medical officer for the government of Bihar.

-Financial Support \& sponsorship: Nil

\section{Author contact information}

1 Department of Health, Government of Bihar, MOBILE - +919955301119/+917677833752, Email drpiyush003@gmail.com

\section{References}

1. Census 2011, available at- https://www.census2011.co.in/census/state/bihar.html visited on-17/06/2021

2. Dr. Piyush Kumar. What Impact Have SARS-CoV-2/Covid-19 Pandemic on the Reproductive and Child Health Programme of Bihar in India over the 3 months after nationwide Lock down announcement in 
March 2020? How SARS-CoV-2 Pandemic era does influence RCH Programme? Immunization? \&nbsp; Maternal Health? Family Planning?, 30 March 2021, PREPRINT (Version 4) available at Research Square [https://doi.org/10.21203/rs.3.rs-348841/v4]

3. Kumar, Dr Piyush, What Are the Factors Responsible for Increase in SARS-CoV-2/COVID-19 Pandemic Related Cases and Death in India in 2021? How Does Environmental, Host \& Agent Factors of Epidemiological Triad Do Influence \& Can Be Utilized to Manage Ongoing Pandemic Cases and Deaths? (April 25, 2021). Available at SSRN: https://ssrn.com/abstract=3833788 or http://dx.doi.org/10.2139/ssrn.3833788

4. Worldometer, available at- https://www.worldometers.info/coronavirus/ visited on-17/06/2021

5. Home Department, Government of Bihar, available at- https://state.bihar.gov.in/home/CitizenHome.html visited on-17/06/2021

6. Verma BK, Verma M, Verma VK, Abdullah RB, Nath DC, Khan HTA, Verma A, Vishwakarma RK, Verma V. Global lockdown: An effective safeguard in responding to the threat of COVID-19. J Eval Clin Pract. 2020 Dec; 26(6):1592-1598. Doi: 10.1111/jep.13483. Epub 2020 Sep 24. PMID: 32970386; PMCID: PMC7719340.

7. Kumar P. Vaccination drive could be affected by frequent change in vaccines, guidelines, policies, interdisciplinary conflicts of medical sciences, mistrust, and evil propaganda over Government data, lack of communication \& health promotion in rural areas of India. [Internet]. OSF Preprints; 2021. Available from: osf.io/pc9uw 